\title{
Layout-Driven Test-Architecture Design and Optimization for 3D SoCs under Pre-Bond Test-Pin-Count Constraint
}

\author{
Li Jiang ${ }^{\dagger *}$, Qiang Xü ${ }^{\dagger *}$ Krishnendu Chakrabarty \\ ${ }^{\dagger}$ Deptartment of CS\&E, The Chinese University of Hong Kong, Shatin, N.T., Hong Kong \\ *CAS-CUHK Shenzhen Institute of Advanced Integration Technology \\ ‡Deptartment of ECE, Duke University, Durham, NC \\ ${ }^{\S}$ Intel Corporation, Santa Clara, CA
}

\begin{abstract}
We propose a layout-driven test-architecture design and optimization technique for core-based system-on-chips (SoCs) that are fabricated using three-dimensional (3D) integration. In contrast to prior work, we consider the pre-bond test-pin-count constraint during optimization since these pins occupy large silicon area that cannot be used in functional mode. In addition, the proposed test-architecture design takes the SoC layout into consideration and facilitates the sharing of test wires between pre-bond tests and post-bond test, which significantly reduces the routing cost for a test-access mechanism in $3 D$ technology. Experimental results for the ITC'02 SoC benchmarks circuits demonstrate the effectiveness of the proposed solution.
\end{abstract}

\section{INTRODUCTION}

As system-on-a-chip (SoC) designs become increasingly complex, interconnects have emerged as the performance and power limiter for giga-scale integrated circuits (ICs). Three-dimensional (3D) technology is able to provide abundant interconnect resources with improved performance and less communication energy by integrating multiple silicon dies with short and dense through-silicon vias (TSVs). As a result, it has become a promising solution to address the interconnect problem [3]. 3D technology also facilitates the integration of disparate technologies such as microelectromechanical systems (MEMS) and various kinds of sensors as they can be fabricated on different silicon layers separately before integration, thereby offering a genuine single-chip system solution. Because of the above benefits, industry experts predict that 3D ICs will occupy a big market share for future semiconductor products [21], despite the still unresolved testing and thermal-management challenges.

In 3D ICs, silicon dies at different layers can be built in three ways: wafer-to-wafer (W2W) bonding [19], die-to-die (D2D) bonding [13], or die-to-wafer (D2W) bonding (for 3D ICs built on two semiconductor wafers only) [4]. Known good dies (KGDs) can be attached through pre-bond wafer-level testing to achieve higher manufacturing yield in D2D bonding or D2W bonding $[3,15]$. Therefore, when the die size is large and/or the defect density is high, they are preferred over $\mathrm{W} 2 \mathrm{~W}$ bonding. In terms of bonding direction, there can be faceto-face bonding or face-to-back bonding. The former allows more interconnects between active devices on different layers but it limits the number of stacked dies to be two; the latter is a scalable solution that supports more stacking layers.

Permission to make digital or hard copies of all or part of this work for personal or classroom use is granted without fee provided that copies are not made or distributed for profit or commercial advantage and that copies bear this notice and the full citation on the first page. To copy otherwise, or republish, to post on servers or to redistribute to lists, requires prior specific permission and/or a fee.

ICCAD'09, November 2-5, 2009, San Jose, California, USA.

Copyright 2009 ACM 978-1-60558-800-1/09/11...\$10.00.
In order to enable pre-bond tests and improve manufacturing yield for 3D ICs, we need to fabricate a number of test pads on the silicon die so that the automatic test equipment (ATE) can probe it during testing. The test pads, however, occupy much larger area compared to the TSVs. Typically, one single test pad can consume area equivalent to hundreds of front-side vias. Therefore, if a large number of test pads are fabricated, the benefits of exploiting TSVs for interconnecting active devices between layers are significantly reduced. As a result, it is essential to take the pre-bond test-pin-count constraint into consideration during test planning. Related prior work in test architecture design and optimization for 3D SoCs [8], however, tries to integrate pre-bond tests and post-bond test together and may lead to high test pad requirement for certain dies.

To tackle the above problem, in this work, we design test architectures for pre-bond tests and post-bond test separately so that the testpin-count constraint can be satisfied in pre-bond tests. By doing so, however, the routing cost for test access mechanisms (TAMs) may be dramatically increased as pre-bond tests and post-bond test have different TAMs. To address this issue, we propose optimization methods that allow us to share routing resources between pre-bond tests and post-bond test as much as possible. Also, we show how to optimize test architectures to further reduce TAM routing cost with little impact on testing time. Experimental results on several 3D adaptations of the ITC' 02 benchmark circuits show that, the proposed layout-driven test architecture design and optimization techniques for 3D SoCs can reduce TAM wire length by up to $50 \%$ with only a small increase in testing time.

The remainder of this paper is organized as follows. Section 2 reviews related work and motivates this paper. The problem investigated in this paper is then formulated in Section 3. Next, our proposed layout-driven test architecture design and optimization techniques are detailed in Section 4. Section 5 presents experimental results to show the advantages of the proposed method. Finally, Section 6 concludes this paper.

\section{PRELIMINARIES AND MOTIVATION}

\subsection{Prior Work in Testing 3D ICs}

Test techniques and design-for-testability (DfT) solutions for 3D ICs are critical issues for the success of 3D technology, as pointed out in $[14,16]$. However, only limited work has been done in this emerging area. Lewis and Lee [12] proposed a scan-island-based design to enable pre-bond tests for incomplete circuits at the architecture level. Wu et al. [18] studied several scan chain design approaches for 3D ICs and compared their routing costs. The above works mainly target 3D ICs that put functional blocks in different silicon layers.

For 3D SoCs with entire embedded cores on different layers, modular testing is an attractive solution as it facilitates the reuse of test patterns. While test architecture design and optimization for twodimensional SoCs have been subject to extensive research [20], these solutions are not readily applicable for testing 3D SoCs. Recently, a test-access mechanism (TAM) optimization technique was proposed 


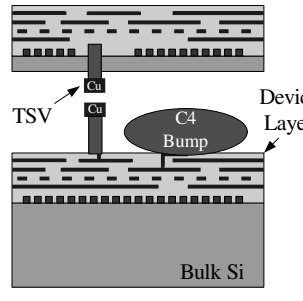

(a)

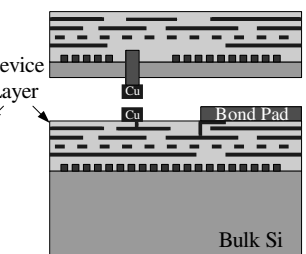

(b)
Figure 1: Pre-bond Test Pad: (a) C4 Bump as Test Pad, (b) WireBond as Test Pad.

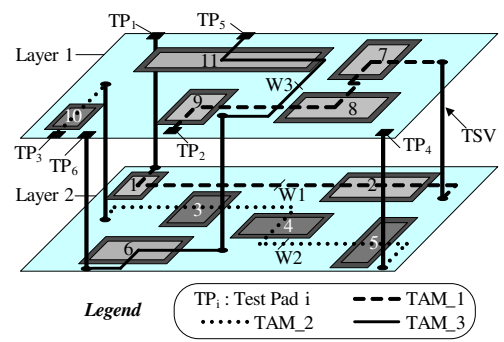

Figure 2: Test architecture for an example 3D SoC.

in [17] to minimize the testing time of 3D SOCs, under limits on the number of TSVs utilized by TAMs. However, pre-bond tests were not considered in this work and hence it can only provide cost-effective solutions for 3D SoCs manufactured with W2W bonding technology. Jiang et al. [8] proposed simulated annealing (SA) based algorithms to optimize modular SoC test architecture considering both pre-bond tests and post-bond test. In this work, the same TAMs that traverse multiple layers in post-bond testing are fully reused for pre-bond tests. Consequently, TAMs can be divided into multiple parts and distributed among the different silicon layers. As all the TAM segments in a particular silicon layer need to be probed during pre-bond testing, a large amount of test pads may be required for those silicon dies that contain many TAM segments. This can be a serious issue in pre-bond testing, as shown in the following section.

\subsection{Test-Pin-Count Constraint}

When conducting pre-bond tests for silicon dies at wafer-level, one of the biggest challenges is how to probe the silicon die effectively. As shown in [1], since fine-grained touchdown probe needles are not available in the next decade, producing dense probe arrays to connect to the ATE is not a viable solution, at least for the near future. Consequently, we have to fabricate test pads ( $\mathrm{C} 4$ bump or wire bond, see Fig. 1) on silicon dies and rely on conventional probing techniques to connect them to the ATE during pre-bond testing [14].

At the same time, however, it is not possible to fabricate a large number of test pads for pre-bond testing in 3D ICs. This is because of the following reasons. According to [1], the pitch for C4 bumps is around $120 \mu \mathrm{m}$, which is much larger than that of TSV $(1.7 \mu \mathrm{m}$ as shown in [22] and this figure keeps shrinking with technology improvements). In other words, one single test pad can consume area equivalent to hundreds of TSVs (see Fig. 1). As these test pads have to be put at the "keep-out area" for TSVs (i.e., TSVs need to keep some distance from any other component), the benefits of exploiting dense TSVs for interconnecting active devices between layers are significantly diminished with the increase of test pads [11]. In 3D technology, except for the bottom layer, the silicon bulks in other layers are thinned for the ease of TSV fabrication. If we conduct pre-bond tests before thinning, we may not be able to detect the failures introduced during the chemical mechanical polishing (CMP) process. If, however, we probe the thinned wafer instead, the probe force (typically $3-10 \mathrm{~g}$ per probe and $60-120 \mathrm{~kg}$ per wafer) during testing becomes a serious concern as these thinned wafers are not mechanically strong enough. Again, it is desired to have less test pads (probes/touchdowns) for silicon dies in pre-bond testing.

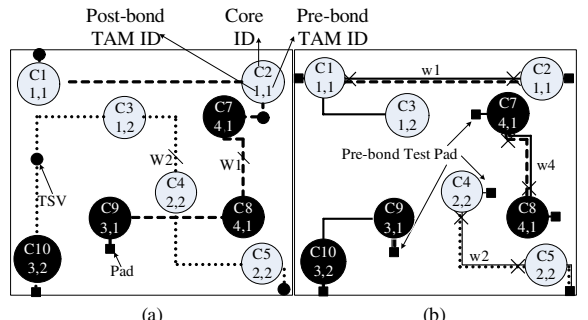

Figure 3: Routing resource sharing example: (a) Test Architecture During Post-bond Test, (b) Reuse TAM During Pre-bond Test.

\subsection{Motivation}

The most straightforward solution to take pre-bond test-pin-count constraint into consideration during the 3D SoC test architecture design and optimization process is to design separate test architectures for pre-bond tests and post-bond test. By doing so, however, the total TAM routing cost for 3D SoCs can be quite high as we have dedicated TAMs for pre-bond tests, resulting in degradation of the chip's routability. As we need to link cores using both pre-bond TAMs and post-bond TAMs and they are used at different times, a natural question is whether we can share some of the routing resources between the two types of TAMs.

We use the following example to demonstrate the possibility of sharing routing resources and its potential benefits. Consider a twolayer 3D SoC containing 11 cores, in which six of them (C1 to $\mathrm{C} 6)$ are on the bottom layer while the other five cores $(\mathrm{C} 7$ to $\mathrm{C} 11)$ are on the top layer. Similar to [8], for the sake of TSV count consideration, we assume a post-bond TAM involved in several layers will route through all cores tested with this TAM on one layer before it goes through TSVs to connect cores in other layers. In this example 3D SoC, three TAMs are used for post-bond testing and they are shown in Fig. 2. As an example, $T A M_{1}$ connects $\mathrm{C} 1, \mathrm{C} 2, \mathrm{C} 7, \mathrm{C} 8$, and $\mathrm{C} 9$ with TAM width $W 1$, starting from test pad $T P_{1}$ and ending at test pad $T P_{2}$.

For the ease of discussion, we map a few cores in the 3D SoC onto one layer as shown in Fig. 3(a). In this figure, each vertex represents a core, in which the upper label is the core ID, while the lower one denotes the pre-bond TAM ID and post-bond TAM ID that this core belongs to. In Fig. 3(b), we show how pre-bond TAMs can reuse the existing test wires for post-bond testing, wherein the solid lines are pre-bond TAMs. It can be easily observed that those wires having both solid and dashed/doted lines can be shared between pre-bond test and post-bond test, which can significantly reduce the total routing cost for TAMs in 3D SoCs. Note that, during pre-bond test, the end points of each TAM are directly routed to deliver test data on its own silicon layer. Here, we assume that these test pad is near the end point, so that we can ignore the distance between end points and test pads.

Obviously, some design-for-testability (DfT) circuitries need to be introduced to enable the routing resource sharing between pre-bond test and post-bond. To be specific, we need: (i) certain multiplexers to select the different test data source for pre-bond test and post-bond test (see the " $\times$ " point shown in Fig. 3(b)); (ii) reconfigurable test wrappers for cores that have different TAM width between pre-bond test and post-bond test (e.g., $[9,10]$ ); (iii) the necessary control mechanisms (typically by introducing extra instructions in test wrapper and JTAG controller).

\section{PROBLEM FORMULATION}

The layout-driven 3D SoC test architecture design and optimization problem investigated in this paper can be formulated as follows:

Problem: Given

- the set of cores $C$ on the 3D SoC, and the test parameters for each core $c \in C$;

- the layout of the 3D SoC, i.e., the physical position of every core $c$, including which layer it sits on and its X-Y coordinate on that layer; 


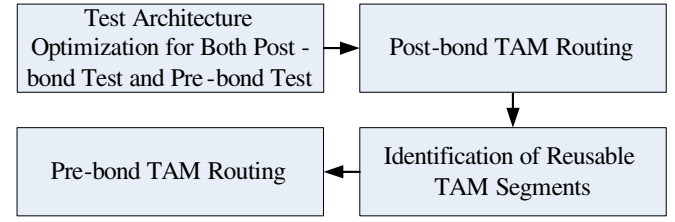

Figure 4: Design Flow For Scheme 1.

- the maximum available TAM width for post-bond test $W_{\text {post }}$;

- the pre-bond test-pin-count constraint $W_{\text {pre }}$;

Our objective is to determine the number of pre-bond TAMs $N_{\text {pre }}$, the number of post-bond TAM $N_{\text {post }}$, the core assignment associated with each TAM $t_{i}$, and the width of each TAMs $W_{t_{i}}$, so that the total testing cost, $C_{\text {total }}=C_{\text {time }} \times \alpha+C_{\text {route }} \times(1-\alpha)$, is minimized ( $\alpha$ is a weighting factor designated by users). Note that $C_{\text {time }}$ and $C_{\text {route }}$ represent the total testing time of the 3D SoC and the total TAM wire length for the $3 \mathrm{D} \mathrm{SoC}$, respectively.

For the case that the pre-bond TAMs and post-bond TAMs are not shared, the routing $\operatorname{cost} C_{\text {route }}$ is simply the total wire length of the two kind of TAMs, that is,

$$
C_{\text {route }}=\sum_{i=0}^{i<N_{\text {pre }}} W_{t_{i}} \times L_{t_{i}}+\sum_{i=0}^{i<N_{\text {post }}} W_{t_{i}} \times L_{t_{i}}
$$

Here, $L_{t_{i}}$ denotes the wire length for TAM $t_{i}$, and we calculate it using the sum of Manhattan distance between adjacent cores in this TAM.

When considering the sharing of pre-bond TAMs and post-bond TAMs, suppose the total length for the shared wires is $C_{\text {reused }}$. The routing cost $C_{\text {route }}$ becomes

$$
C_{\text {route }}=\sum_{i=0}^{i<N_{\text {pre }}} W_{t_{i}} \times L_{t_{i}}+\sum_{i=0}^{i<N_{\text {post }}}\left(W_{t_{i}} \times L_{t_{i}}\right)-C_{\text {reused }}
$$

It is worth noting that test wrapper design and optimization is a subproblem of the above problem, and we use the algorithms in [7, 9] to optimize the IEEE Std. 1500-compliant test wrapper and the reconfigurable test wrapper, respectively.

\section{LAYOUT-DRIVEN TEST ARCHITECTURE DESIGN AND OPTIMIZATION}

We address the above problem progressively in this section, denoted as Scheme 1 and Scheme 2, respectively. In Scheme 1, we consider the case that test architectures for both pre-bond tests and post-bond test are fixed, and we propose a greedy heuristic to share test wires between pre-bond TAM and post-bond TAM as much as possible. In Scheme 2, to further reduce routing cost, we consider flexible prebond test architecture while keeping the post-bond test architecture and its TAM routing unchanged, and then we optimize the pre-bond test architecture so that the total cost $C_{\text {total }}$ is minimized. The reason behind the above strategy is: (i) making both pre-bond test architecture and post-bond test architecture flexible would lead to an extremely large solution space, and hence it is rather difficult to find a good solution within limited computational time; (ii) making pre-bond test architecture (instead of the post-bond test architecture) flexible has the benefit that it only affects the routing in one layer.

\subsection{Scheme 1: TAM Wire Reuse with Fixed Test Architectures}

The design flow for this scheme is shown in Fig. 4. Firstly, we optimize the test architecture for both pre-bond tests and post-bond test, which gives us the number of TAMs, the width for each TAM, and the cores tested on each TAM for each kind of test. Then, we take the 3D SoC layout into consideration and conduct post-bond TAM routing. Next, we identify reusable TAM segments out of the post-bond TAM and conduct pre-bond TAM routing to share them as much as possible. We elaborate the above procedures in detail in the following.

\subsubsection{Post-bond TAM Routing}

Given a post-bond TAM with several cores tested on it, we first map these cores belonging to different layers onto one virtual layer (as shown in Section 2.3). For the connections that link two cores on different layers, we can ignore the routing cost for the TSVs due to its short length. Given the test architecture, we are to route all the cores belonging to the same TAM sequentially, and hence the routing problem is equivalent to the NP-Hard Traveling Salesman (TSP) problem [5].

To tackle this problem, we first construct a complete graph for all the cores (vertices) belonging to the TAM and the weight of each edge represent its routing cost (i.e., the distance between the two cores multiply the TAM width) between the linked two cores (denoted as $\mathcal{S G}$, see Fig. 5(a)). We have another acyclic graph used to store the final routing result (initialized with no edges), denoted as $\mathcal{E} \mathcal{G}$. We consider all the edges in $S \mathcal{G}$ as candidate TAM segments and gradually build $\mathcal{E} G$, using the algorithm shown in Fig. 6.

The input to the post-bond TAM routing algorithm is a set of cores which belong to a TAM, and the output is a core sequence indicating the routing order for the cores on this TAM and its routing cost. In lines 1-4, we construct the completed graph $\mathcal{S} G$ and assign the weight $\left(L_{t i} \times W_{t i}\right)$ on them. In line 5, we sort all the edges according to their weights. Then, in every iteration (lines 6-10), we move edges from $\mathcal{S G}$ to $\mathcal{E} G$ in a greedy manner (i.e., we move the edge with the smallest weight), e.g., the solid line A-B with length 1 in Fig. 5(b) and the solid line B-C with length 3 in Fig. 5(c)). As the procedure going, more edges (i.e., TAM segments) are moved into $\mathcal{E} \mathcal{G}$ and they are gradually linked together as a path (e.g., the linked path A-B-C in Fig. 5(c)). It is important to note that, there are two kinds of redundant edges that should be deleted in every iteration after a new edge is moved into $\mathcal{E} \mathcal{G}$ (line 10): (i) Any edge should be deleted if either of its vertex is an internal vertex (all vertices except two end points of a path) in current $\mathcal{E} \mathcal{G}$; (ii) Any edge that would generate a cycle in $\mathcal{E} \mathcal{G}$ should be deleted since $\mathcal{E} \mathcal{G}$ should be acyclic all the time. Taking Fig. 5(c) as an example, edge B-E and B-D are the first kind of redundant edges, since vertex $\mathrm{B}$ is a internal vertex. Edge $\mathrm{A}-\mathrm{C}$ is the second kind of redundant edge, since A-B-C-A will become a cyclic graph if we move A-C into $\mathcal{E} G$. Finally, all the paths are linked together to form one single path (e.g., solid lines A-B-C-D-E in Fig. 5(d)), which gives us the final TAM routing order and its cost, as returned from lines 11-12.

\subsubsection{Reuse Strategy for TAM Routing Resources}

Given the TAM routing for post-bond test, our problem now is to route the pre-bond TAM in such a manner that we can reuse the postbond TAM test wires as much as possible. To reduce the problem complexity, we first divide every TAM into a set of TAM segments, each linking two adjacent cores on the same silicon layer belonging to the TAM. After routing all the post-bond TAMs, every post-bond TAM segment is considered to be reusable by any pre-bond TAM segment that is on the same silicon layer. For the sake of simplicity, we consider each TAM segment of pre-bond test can reuse test wires from only one TAM segment of post-bond test, and each TAM segment of postbond test can be reused by only one TAM segment of pre-bond test. It should be noted that, we have excluded those TAM segments that link two cores on different layers.

We examine several scenarios to illustrate how we can reuse postbond TAM routing resources for pre-bond tests (see Fig. 7). With

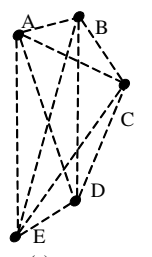

(a)

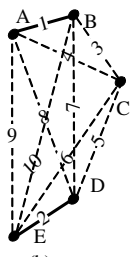

(b)

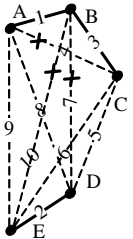

(c)

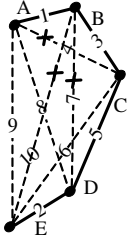

(d)
Figure 5: An Example of Post-Bond TAM Routing. 


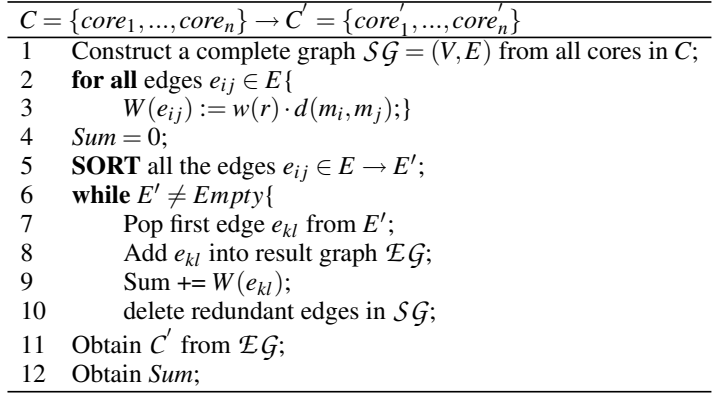

Figure 6: Post-Bond TAM Routing Algorithm

given core layout position (modeled as the center point of the cores), we can draw a bounding rectangle for each TAM segment as shown in Fig. 7(a). To connect these two cores, we can have any routes within this bounding rectangle as long as there is no detour (e.g., route A, B or C), and the Manhattan distance of these routes (i.e., the half perimeter of the bounding rectangle) are all the same.

Let us consider the 3D SoC example demonstrated in Fig. 2. TAM segments $\mathrm{C} 1-\mathrm{C} 2$ and $\mathrm{C} 3-\mathrm{C} 4$ are from post-bond TAM 1 and TAM 3 (see Fig. 7(b)-(d)). Fig. 7(b) depicts the case that a pre-bond TAM segment $\mathrm{C} 1-\mathrm{C} 3$ needs to be routed; while Fig. 7(c) presents another case that we need to route another pre-bond TAM segment C3-C4.

Considering the bounding rectangles for TAM segments discussed above, it is clear that the coincided rectangle is where we can route the pre-bond TAM that reuses post-bond TAM routing resources (i.e., the grey parts in Fig. 7(b)-(d)). It is important to note that, however, the reusable wire length is not always the half perimeter of the coincided rectangle (see Fig. 7(d)), and it is calculated as follows.

Let us denote the slope of diagonal line with its two end points placed from up-left to bottom-right as negative (e.g., C1-C2 of Fig. 7(b)(d), C1-C3 of Fig. 7(b)-(c) and C4-C3 of Fig. 7(c)). On the contrary, the slope of diagonal line with its two end points placed from up-right to bottom-left is positive (C3-C4 of Fig. 7(d)). Considering two TAM segments that share test wires, if the slopes of their diagonal lines are the same (i.e., all negative or all positive), as shown in Fig. 7(b)-(c), the reusable wire length is half perimeter of coincided rectangle. If the slopes are different (i.e., one is negative while the other is positive), however, the reusable wire length is the longer edge of the coincided rectangle, as shown in Fig. 7(d).

In view of the above discussion, the problem of reusing post-bond TAM routing resources in a pre-bond TAM cane be stated in terms of how to combine the one-to-one pairs of TAM segments (one from pre-bond test and the other from post-bond test) so that the total routing cost is minimized. We propose a greedy heuristic to solve this problem, as described next.

\subsubsection{Greedy Heuristic for Pre-Bond TAM Routing}

Fig. 8 presents our proposed greedy heuristic for pre-bond TAM routing, which tries to reuse the routing resources of post-bond TAMs as much as possible.

In line 1, we acquire the possible reusable TAM segments for postbond test. Similar to the post-bond TAM routing algorithm shown in Fig. 6, we move the edge with the lowest routing cost from $S \mathcal{G}$ to $\mathcal{E} G$ iteratively in a greedy manner. In lines $2-4$, we construct a completed graph for every TAM for pre-bond test in the layer, and put
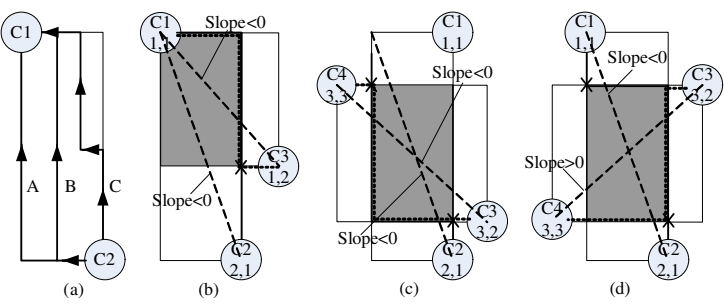

Figure 7: Reusable Routing Resources Represented by Bounding Rectangle.

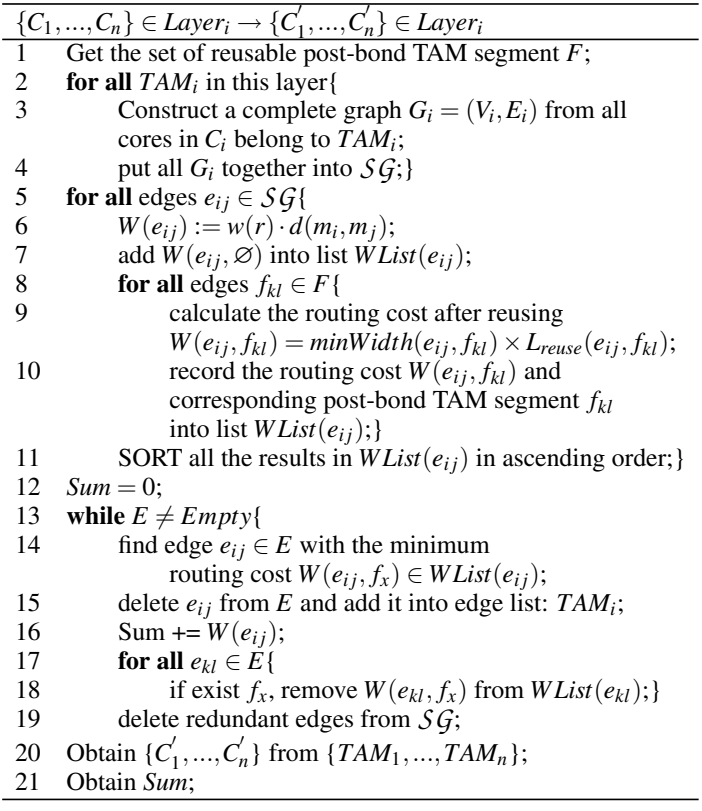

Figure 8: Greedy Heuristic for Pre-Bond TAM Routing

all these complete graphs together into $\mathcal{S} \mathcal{G}$. The reason behind this is that a reusable post-bond TAM segment can be a reusable candidate for TAM segments from more than one pre-bond TAMs. Since each TAM segment of pre-bond test has more than one reusable candidates and each reusable candidates can only be reused at most once, we build a list for each TAM segment of pre-bond test, and store all possible reusable candidates into the list (lines 8-10). To be specific, if one edge cannot reuse any of the TAM segments of post-bond test in that layer, we simply use the original routing cost, calculated by the wire length multiplied by its TAM width, and add it into the list (lines 6-7). If it has a reusable candidate, its routing cost is updated accordingly. In addition, the TAM widths can be different between pre-bond TAM and post-bond TAM. We choose the smaller one to calculate the routing cost of reused wires by multiplying it with the reused wire length. And then we use the original routing cost minus the routing cost of reused wire as the new routing cost of this edge (line 9).

Here, we maintain the list for each edge to keep all the possible reusable TAM segments, and their corresponding updated routing costs (line 10). After sorting the list according to their routing cost (line 11), the head item of each list is the edge with the least routing cost (either with or without reuse strategy). In every iteration, we choose the edge with least routing cost (i.e. value of head item in the list linking to this edge) and move it into $\mathcal{E} \mathcal{G}$ (lines 12-15). Since every reusable candidate can only be reused for at most once, we delete this reused segment from all other edges in $S \mathcal{G}$ (lines 17-18). Finally, we obtain the routing result and its cost (lines 19-21).

We take an example extended from Fig. 3 to elaborate the above process. After constructing the complete graph from all cores in prebond test, we obtain the list of TAM segments shown in Fig. 9, which keeps all possible reusable post-bond TAM segment in the list of every pre-bond TAM segment. For example, for segment [TAM1(C2,C3)], there are two reusable candidates in its list, that is, post-bond TAM segment $(\mathrm{C} 1, \mathrm{C} 2)$ and $(\mathrm{C} 3, \mathrm{C} 4)$, The corresponding routing costs are 3 and 10 , respectively. The original routing cost is 18 . In this example, we pick up the pre-bond TAM segment [TAM1 $(\mathrm{C} 1, \mathrm{C} 2)]$ first since it has the minimum routing cost 0 by reusing the post-bond TAM segment $[0(\mathrm{C} 1, \mathrm{C} 2)]$. We then move [TAM1 $(\mathrm{C} 1, \mathrm{C} 2)]$ from $S \mathcal{G}$ to the $\mathcal{E} G$. Afterwards, the reusable post-bond TAM segments $[(\mathrm{C} 1, \mathrm{C} 2)]$ are deleted from all the lists. Then, in the second iteration, [TAM2(C4,C5)] is chosen, and the reusable post-bond TAM segment $[(\mathrm{C} 4, \mathrm{C} 5)]$ is deleted. Next, we know that $[\mathrm{TAM} 1(\mathrm{C} 1, \mathrm{C} 3)]$ has the minimum routing cost of 8 without any reuse. Again, it is moved to $\mathcal{E} \mathcal{G}$. At last, we delete the redundant segment [TAM1 $(\mathrm{C} 2, \mathrm{C} 3)]$. 


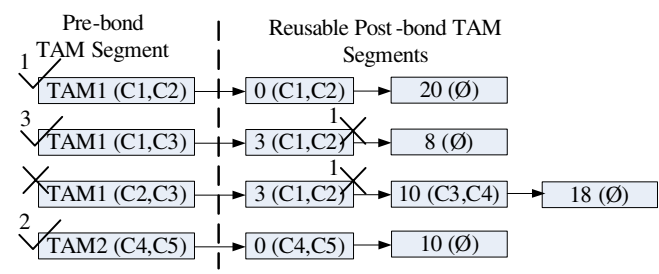

Figure 9: Example for the Proposed Algorithm to Reuse TAM Routing Resources.

\subsection{Scheme 2: TAM Wire Reuse with Flexible Pre-bond Test Architecture}

By changing the test architecture for pre-bond tests, we can further reduce their routing cost as it is possible to share more routing resources from post-bond TAM. However, it may lead to the increase of testing time, since we change the test architecture which can previously lead to a near optimal solution in terms of testing time cost. As a result, we would like to sacrifice only limited testing time to obtain much better routing cost. In order to achieve the above objective, we extend the simulated annealing-based 3D SoC test architecture optimization procedure presented in [8]. Our design flow for Scheme 2 is shown in Fig. 10.

Similar to [8], the optimization procedure is comprised of two parts: the outer SA-based core assignment and the inner heuristic-based TAM width allocation. In the SA-based core assignment procedure, cores are randomly moved between multiple TAMs according to certain rules to give a core assignment solution. Note that, the SA procedure guarantees that we are able to reach all possible core assignment solutions with limited number of movements. Then, for each solution, the heuristic-based TAM width allocation procedure is called (see Fig.11), which starts from the initial solution (line 1) and iteratively tries to find the TAM that leads to the lowest total test cost after assigning one-bit wire (line 5-12). In line 7, we use proposed greedy reusable heuristic (as shown in Fig. 8) to calculate its routing cost, and obtain the total test cost including both routing cost and testing time cost (line 8). If this one-bit TAM wire can result in cost reduction, we will allocate it in this iteration (line 13-15). If not, we increase the width of the to-be-assigned TAM wire by one-bit and try next iteration without any allocation (line 16-17), until a lower cost is found. Eventually, we obtain the pre-bond TAM design with the least test cost (see Fig. 10). It should be noted that the optimization for post-bond test architecture only needs to be done once in the whole procedure in Fig. 10. The same as reusable TAM segment identification is. There is a global variable namely temperature related to the acceptance probabilities of each solution. It is set to be a large value when the simulated annealing procedure starts, and gradually decreases. Finally, the simulated annealing procedure terminates when the temperature is lower than a threshold.

\section{EXPERIMENTAL RESULTS}

\subsection{Experimental Setup}

To demonstrate the effectiveness of the proposed layout-driven 3D SoC test architecture design and optimization technique, we present experimental results for four revised ITC'02 benchmark SoCs (p22810, p34392, p93791, and t512505). We map these SoCs onto three silicon layers randomly and try to balance the total area of each layer, in

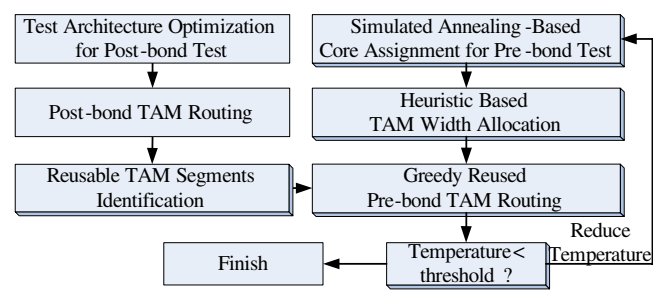

Figure 10: Design Flow for Scheme 2.

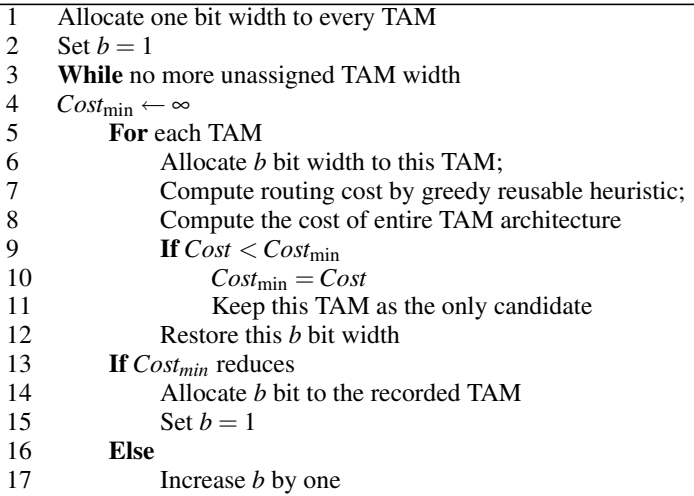

Figure 11: Heuristic-based TAM Width Allocation.

which the area for a core is estimated based on the number of internal inputs/outputs and scan cells (if any). An academic floorplanner is then utilized to get the coordinates for each core, which are used for wire length calculation.

Test Bus architecture is assumed in our experiments. The pre-bond TAM width is fixed to be 16 by taking the test-pin-count constraint into consideration. We compare three kinds of test architecture design and optimization solutions. The first one (denoted as No Reuse), implemented the algorithm in [6] to optimize testing time and it uses the TAM routing algorithm shown in Fig. 6 to route both post-bond TAMs and pre-bond TAMs without sharing routing resources between the two kinds of TAMs. The second one (denoted as Reuse), resorts to the same heuristic to optimize testing time as No Reuse, but uses the greedy heuristic algorithm shown in Fig. 8 to route pre-bond TAMs. The last scheme (denoted as $S A$ ), has the same procedures as Reuse to optimize post-bond testing time and post-bond TAM routing, and it uses the SA-based optimization procedure shown in Section 4.2 to adjust the pre-bond test architectures for further test cost reduction. We do not compare against [8], because it does not take test-pin-count constraint into consideration and it may result in pre-bond test architecture with more than 16-bit TAMs, preventing fair comparison.

\subsection{Results and Discussion}

As can be observed from Tables 1, the testing time of No Reuse scheme and Reuse scheme (i.e., Scheme 1) are the same as they employ the same test architecture (with different routing strategies only). In most cases, $S A$ scheme (i.e., Scheme 2) slightly increase the prebond testing time since it sacrifices some testing time to achieve reduced routing cost, but no more than $1 \%$ or $2 \%$ except for $\mathrm{p} 34392$ with large post-bond TAM width. In very few cases, both the testing time and the routing cost for the $S A$ scheme are the smallest among the three schemes (e.g., p34392 with TAM width 40).

The routing cost reduction brought by the greedy TAM reusing algorithm used in Reuse scheme is considerable when comparing with No Reuse scheme. The reduction ratio can be as high as $-21.23 \%$ for p34392 with TAM width 40 . With flexible pre-bond test architecture used in the $S A$ scheme, the savings in routing cost is even larger, in the range between $-24.87 \%$ and $-49.39 \%$. The average routing reduction is around $33 \%, 38 \%, 46 \%$, and $28 \%$ for the four benchmark SoCs p93791 produces better results because there is no stand-out large core in this SoC, which can serve as a bottleneck during the optimization process. By contrast, $\mathrm{t} 512505$ has a large core that alone requires a large post-bond TAM width on its own, which essentially reduces the reusable TAM segments.

Generally speaking, with the growth of post-bond TAM width from 16 to 64 , the routing cost reduction ratio increases in the beginning and drops at the end. The main reason is that, when post-bond TAM width grows, the width of reusable TAM segments also goes up; while the TAM width keeps to be 16 for pre-bond tests, the demanded reusable TAM width does not increase, leaving more reusable TAM width idle.

Finally, we present the layout of one layer in 3D SoC p93791 to demonstrate the effectiveness of our TAM reuse strategy (see Fig. 12). 


\begin{tabular}{|c|c|c|c|c|c|c|c|c|c|c|c|c|c|c|c|c|c|c|}
\hline \multirow{2}{*}{$\begin{array}{l}\text { Width } \\
\text { (bit ) }\end{array}$} & \multicolumn{3}{|c|}{ Total Testing Time } & \multirow{2}{*}{$\begin{array}{l}\text { Ratio } \\
\Delta^{T}(\%) \\
\end{array}$} & \multicolumn{3}{|c|}{ Routing Cost } & \multicolumn{2}{|c|}{ Ratio } & \multicolumn{3}{|c|}{ Total Testing Time } & \multirow{2}{*}{\begin{tabular}{|c|} 
Ratio \\
$\Delta^{T}(\%)$ \\
\end{tabular}} & \multicolumn{3}{|c|}{ Routing Cost } & \multicolumn{2}{|c|}{ Ratio } \\
\hline & No Reuse & Reuse & SA & & No Reuse & Reuse & SA & $\Delta_{1}^{W}(\%)$ & $\Delta_{2}^{W}(\%)$ & NoReuse & \begin{tabular}{|l|} 
Reuse \\
\end{tabular} & SA & & No Reuse & Reuse & SA & $\Delta_{1}^{W}(\%)$ & $\Delta_{2}^{W}(\%)$ \\
\hline & \multicolumn{9}{|c|}{ p22810 } & \multicolumn{9}{|c|}{ p34392 } \\
\hline 16 & 948714 & 948714 & 959753 & 1.16 & 18677 & 15922 & 12648 & -14.7 & -32.3 & 2100312 & 2100312 & 2088962 & -0.54 & 15154 & 13595 & 7760 & -10.29 & -48.79 \\
\hline 24 & 730866 & 730866 & 738800 & 1.09 & 19549 & 17744 & 14567 & -9.23 & -25.5 & 1802341 & 1802341 & 1791078 & -0.62 & 27624 & 22766 & 17422 & -17.59 & -36.93 \\
\hline 32 & 647043 & 647043 & 653060 & 0.93 & 17289 & 15445 & 10898 & -10.6 & -36.9 & 1592668 & 1592668 & 1595286 & 0.16 & 25220 & 20946 & 13949 & -16.95 & -44.69 \\
\hline 40 & 608139 & 608139 & 611087 & 0.48 & 14547 & 11826 & 8240 & -18.7 & -43.4 & 1579078 & 1579078 & 1538795 & -2.55 & 43384 & 34172 & 24439 & -21.23 & -43.67 \\
\hline 48 & 584380 & 584380 & 587328 & 0.50 & 25532 & 23402 & 19181 & -8.34 & -24.9 & 1572840 & 1572840 & 1567728 & -0.33 & 33544 & 28460 & 23705 & -15.16 & -29.33 \\
\hline 56 & 562561 & 562561 & 563882 & 0.23 & 17138 & 15739 & 10754 & -8.16 & -37.3 & 1571728 & 1571728 & 1819571 & 15.77 & 34068 & 29669 & 23423 & -12.91 & -31.25 \\
\hline \multirow[t]{2}{*}{64} & 550549 & 550549 & 553497 & 0.54 & 17642 & 16548 & 11320 & -6.20 & -35.8 & 1571728 & 1571728 & 1758060 & 11.86 & 34068 & 29669 & 23145 & -12.91 & -32.06 \\
\hline & \multicolumn{9}{|c|}{ p93791 } & \multicolumn{9}{|c|}{ t512505 } \\
\hline 16 & 3631177 & 3631177 & 3726714 & 2.63 & 35709 & 32674 & 18803 & -8.5 & -47.34 & 23494872 & 23494872 & 23494872 & \begin{tabular}{l|l}
0 &
\end{tabular} & 2693 & 2409 & 1974 & -10.55 & -26.70 \\
\hline 24 & 2782827 & 2782827 & 2791223 & 0.30 & 42034 & 36818 & 23727 & -12.41 & -43.55 & 23417347 & 23417347 & 23494872 & 0.33 & 2687 & 2403 & 1968 & -10.57 & -26.76 \\
\hline 32 & 2347166 & 2347166 & 2390750 & 1.86 & 41403 & 38383 & 20953 & -7.29 & -49.39 & 18232745 & 18232745 & 18310270 & 0.43 & 1723 & 1441 & 1004 & -16.37 & -41.73 \\
\hline 40 & 2099123 & 2099123 & 2153380 & 2.58 & 44550 & 40509 & 24709 & -9.07 & -44.54 & 18192297 & 18192297 & 18438359 & 1.35 & 2721 & 2405 & 2002 & -11.61 & -26.42 \\
\hline 48 & 1932476 & 1932476 & 1946263 & 0.71 & 43546 & 37730 & 22749 & -13.36 & -47.76 & 18192297 & 18192297 & 18352952 & 0.88 & 2721 & 2405 & 2002 & -11.61 & -26.42 \\
\hline 56 & 1814195 & 1814195 & 1842030 & 1.53 & 61754 & 54135 & 33205 & -12.34 & -46.23 & 18192297 & 18192297 & 18269822 & 0.43 & 2721 & 2405 & 2002 & -11.61 & -26.42 \\
\hline 64 & 1708376 & 1708376 & 1737586 & 1.71 & 52686 & 46992 & 26974 & -10.81 & -48.80 & 18192297 & 18192297 & 18482093 & 1.59 & 2721 & 2405 & 2002 & -11.61 & -26.42 \\
\hline
\end{tabular}

Table 1: Experimental Results for 3D SoC p22810 and p34392.

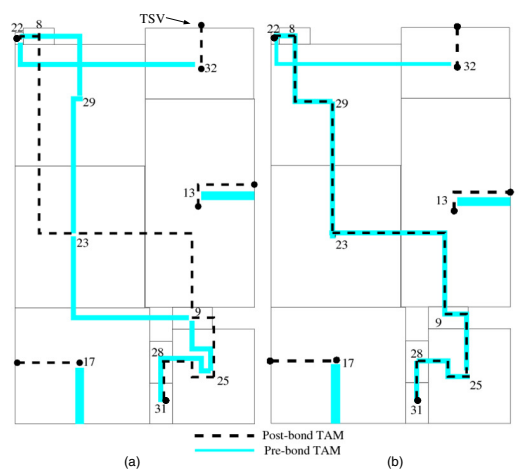

Figure 12: Pre-Bond TAM Routing in p93791. (a) without Reusing Post-Bond TAMs; (b) Reusing Post-Bond TAMs.

The TAM segments of post-bond TAM on this layer is shown as dashed lines; while the solid lines are the pre-bond TAMs in this layer. Note that, if a TAM only goes through one single core in this layer, it cannot be reused for pre-bond TAM (e.g., the one for cores 13). Fig. 12(a) depicts the test wires without reusing any post-bond TAM segments. With our reuse methodology as shown in Fig. 12(b), we can see that the the routing overhead for TAMs can be significantly reduced.

\section{CONCLUSION}

In 3D technology, test pads occupy a much larger area when compared to TSVs and hence we can only fabricate a limited number of test pads for pre-bond testing. In contrast to prior work that does not take such pre-bond test-pin-count constraint into consideration during the 3D SoC test-architecture design and optimization process, we design dedicated pre-bond and post-bond test architectures to satisfy the given test pad constraint. Then, we present novel layout-driven optimization techniques to share the TAM routing resources between pre-bond tests and post-bond test, which can significantly reduce TAM routing cost with little impact on testing time, as shown in our experimental results for ITC'02 SoC benchmark circuits.

\section{ACKNOWLEDGEMENTS}

This work was supported in part by the General Research Fund CUHK417406, CUHK417807, and CUHK418708 from Hong Kong SAR Research Grants Council (RGC), in part by National Science Foundation of China (NSFC) under grant No. 60876029, in part by a grant N CUHK417/08 from the NSFC/RGC Joint Research Scheme, and in part by the National High Technology Research and Development Program of China (863 program) under grant no. 2007AA01Z109.

\section{REFERENCES}

[1] International Technology Roadmap for Semiconductors (ITRS). 2009. Available: www.itrs.net, 2009

[2] K. Banerjee, et al. 3-D ICs: A Novel Chip Design for Improving Deep-Submicrometer Interconnect Performance and Systems-on-Chip Integration. In Proc. of the IEEE, 89(5):602-633, 2001.

[3] W. R. Davis, et al. Demystifying 3D ICs: The Pros and Cons of Going Vertical IEEE Design\& Test, pp. 498-510, 2005.

[4] T. Fukushima, et al. New Three-Dimensional Integration Technology Using Chip-to-Wafer Bonding to Achieve Ultimate Super-Chip Integration. Japanese Journal of Applied Physics, 45(4B):3030, 2006.

[5] S. Goel and E. Marinissen. Layout-driven SOC Test Architecture Design for Test Time and Wire Length Minimization. In Proc. DATE, pp. 738-743, 2003.

[6] S. K. Goel and E. J. Marinissen. SOC Test Architecture Design for Efficient Utilization of Test Bandwidth. ACM Transactions on Design Automation of Electronic Systems, 8(4):399-429, Oct. 2003.

[7] V. Iyengar, K. Chakrabarty, and E. J. Marinissen. Co-Optimization of Test Wrapper and Test Access Architecture for Embedded Cores. Journal of Electronic Testing: Theory and Applications, 18(2):213-230, Apr. 2002.

[8] L. Jiang, L. Huang, and Q. Xu. Test Architecture Design and Optimization for Three-Dimensional SoCs. In Proc. DATE, pp. 220-225, 2009.

[9] S. Koranne. Design of Reconfigurable Access Wrappers for Embedded Core Based SoC Test. IEEE Transactions on Very Large Scale Integration (VLSI) Systems,11(5):955-960, 2003.

[10] E. Larsson and Z. Peng. A Reconfigurable Power-Conscious Core Wrapper and it Application to SOC Test Scheduling. In Proc. ITC, pp. 1135-1144, 2003.

[11] H.-H. S. Lee and K. Chakrabarty. Test Challenges for 3D Integrated Circuits. In Special Issue on 3D IC Design and Test. DATE, 2009.

[12] D. L. Lewis and H.-H. S. Lee. A Scan-Island Based Design Enabling Pre-Bond Testability in Die-Stacked Microprocessors. In Proc. ITC, pp. 1-8, 2007.

[13] G. H. Loh, Y. Xie, and B. Black. Processor Design in 3D Die-Stacking Technologies. IEEE Micro, 27(3):31-48, 2007.

14] T. M. Mak., et al. Testing of 3D Circuites. In Handbook of 3D Integration:Technology and Applications using 3D Integrated Circuits., Wiley-CVH, 2008.

[15] G. Smith, et al. Yield Considerations in the Choice of 3D Technology. In Proc.ISSM, pp. 1-3, 2007.

[16] T. Vucurevich. The Long Road to 3-D Integration: Are We There Yet? Keynote speech at the 3D Architecture Conference, 2007.

[17] X. Wu, et al. Test-Access Mechanism Optimization for Core-Based Three-Dimensional SOCs. In Proc. ICCD, 2008

[18] X. Wu, P. Falkenstern, and Y. Xie. Scan Chain Design for Three-Dimensional Integrated Circuits (3D ICs). In Proc. ICCD, pp. 208-214, 2007.

[19] Y. Xie, et al. Design Space Exploration for 3D Architectures. ACM Journal on Emerging Technologies in Computing Systems (JETC), 2(2):65-103, 2006

[20] Q. Xu and N. Nicolici. Resource-Constrained System-on-a-Chip Test: A Survey. IEE Proc., Computers and Digital Techniques, 152(1):67-81, January 2005.

[21] Yole Development. Market Trends for 3D Stacking. www.yole.fr.

[22] L. Zhou, C. Wakayama, and C. Shi. CASCADE: A Standard Supercell Design Methodology With Congestion-Driven Placement for Three-Dimensional Interconnect-Heavy Very Large-Scale Integrated Circuits. IEEE Transaction on Computer Aided Design of Integrated Circuits, 26(7):1270-1282, 2007. 